\title{
Pyroelectric Nanogenerators for Driving Wireless Sensors
}

\author{
Ya Yang, ${ }^{\dagger, \S}$ Sihong Wang, ${ }^{\dagger, \S}$ Yan Zhang, ${ }^{\ddagger}$ and Zhong Lin Wang*, ${ }^{\dagger}$, \\ ${ }^{\dagger}$ School of Materials Science and Engineering, Georgia Institute of Technology, Atlanta, Georgia 30332-0245, United States \\ ${ }^{\ddagger}$ Beijing Institute of Nanoenergy and Nanosystems, Chinese Academy of Sciences, Beijing, China
}

\section{Supporting Information}

ABSTRACT: We demonstrate a pyroelectric nanogenerator (PENG) based on a lead zirconate titanate (PZT) film, which has a pyroelectric coefficient of about $-80 \mathrm{nC} / \mathrm{cm}^{2} \mathrm{~K}$. For a temperature change of $45 \mathrm{~K}$ at a rate of $0.2 \mathrm{~K} / \mathrm{s}$, the output open-circuit voltage and short-circuit current density of the PENG reached $22 \mathrm{~V}$ and $171 \mathrm{nA} / \mathrm{cm}^{2}$, respectively, corresponding to a maximum power density of $0.215 \mathrm{~mW} /$ $\mathrm{cm}^{3}$. A detailed theory was developed for understanding the high output voltage of PENG. A single electrical output pulse

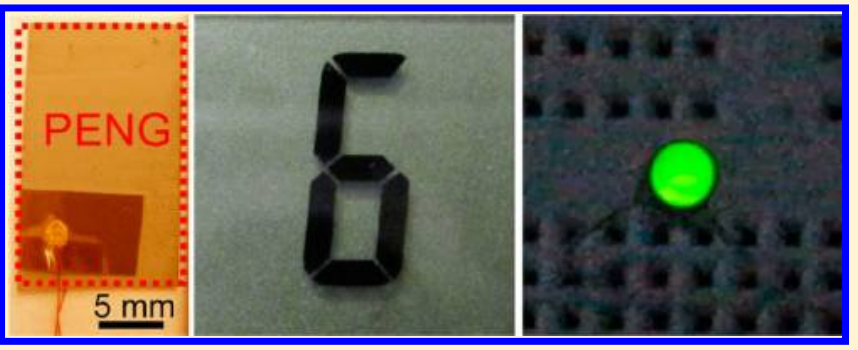
can directly drive a liquid crystal display (LCD) for longer than $60 \mathrm{~s}$. A Li-ion battery was charged by the PENG at different working frequencies, which was used to drive a green lightemitting diode (LED). The demonstrated PENG shows potential applications in wireless sensors.

KEYWORDS: Pyroelectric effect, nanogenerators, PZT, self-powered nanosystems

$\mathrm{O}$ wing to a tremendously increased energy consumption of modern societies, the development of green and renewable nanoenergy sources is becoming one of the most important fields of research. ${ }^{1-3}$ There are several typical physics effects that can be used to fabricate nanogenerators (NGs) for harvesting energy from the ambient environment. Both piezoelectric and triboelectric effects can be used to harvest mechanical energy. ${ }^{4-7}$ The pyroelectric effect is based on the change of spontaneous polarization in certain anisotropic solids due to the temperature fluctuation, ${ }^{8}$ which can be used to harvest thermal energy from the change in temperature. Although the Seebeck effect can also be used to harvest thermal energy by utilizing a temperature difference between two ends of the device for driving the diffusion of charge carriers, 9,10 the pyroelectric effect has to be the choice in an environment where the temperature is spatially uniform but time-dependent. ${ }^{11}$

Self-powering nanotechnology has been developed since 2005 with the aim to build self-powered systems that can operate independently and wirelessly without the use of a battery or other energy storage/supply systems. ${ }^{12-14}$ We have been developing nanogenerators for this purpose. One of the great applications for the self-powered system is to use nanogenerators to drive personal electronics, such as LCDs and LEDs. Currently, piezoelectric NGs have been used to drive some electrical devices by harvesting mechanical energy from the environment. ${ }^{14}$ Although different designs of pyroelectric nanogenerators (PENGs) have been reported, the output voltage and current of these devices are still very low (voltage below $0.1 \mathrm{~V}$, and current below $1 \mathrm{nA}$ ), ${ }^{11,15}$ which are not enough for driving any commercial electronics. To solve this problem, the performance optimization of the PENGs is desperately needed. Here, we demonstrated a lead zirconate titanate (PZT) film PENG, where the output open-circuit voltage and short-circuit current density can be up to $22 \mathrm{~V}$ and $171 \mathrm{nA} / \mathrm{cm}^{2}$, respectively. Under a change in temperature of 45 $\mathrm{K}$, a single output pulse of PENG can continuously drive a LCD for longer than $60 \mathrm{~s}$. A Li-ion battery can be charged by such PENGs under different frequencies.

Our design of the PENG is based on the change in spontaneous polarization of a PZT thin film due to the timedependent temperature fluctuation to drive electrons to flow in external circuit. The detailed fabrication method of the PENG is given in the Experimental Section. Figure la shows an optical image of the fabricated PENG, indicating that the device has a length of $21 \mathrm{~mm}$ and a width of $12 \mathrm{~mm}$. Figure $1 \mathrm{~b}$ shows a cross-sectional scanning electron microscopy (SEM) image of the device, revealing the thickness of the device to be $175 \mu \mathrm{m}$. The large size PZT microfilm was chosen for easy manipulation. The same principle and methodology can be applied to the PZT nanofilm. The enlarged SEM image of the cross section indicates that the film consists of a large number of crystal grains, as shown in Figure 1c.

The output voltage and current of the PENG have been measured by varying the temperature in the vicinity of the NG from 295 to 299 K, as shown in Figure 1d. The corresponding differential curve of the change in temperature with time shows that the peak value of the temperature changing rate is about $0.2 \mathrm{~K} / \mathrm{s}$. Under the forward connection, a sharp negative voltage/current pulse $(\sim 2.8 \mathrm{~V} ; 42 \mathrm{nA})$ was observed when the temperature was quickly increased from 295 to 299 K (Figure

Received: October 9, 2012

Revised: October 30, 2012 


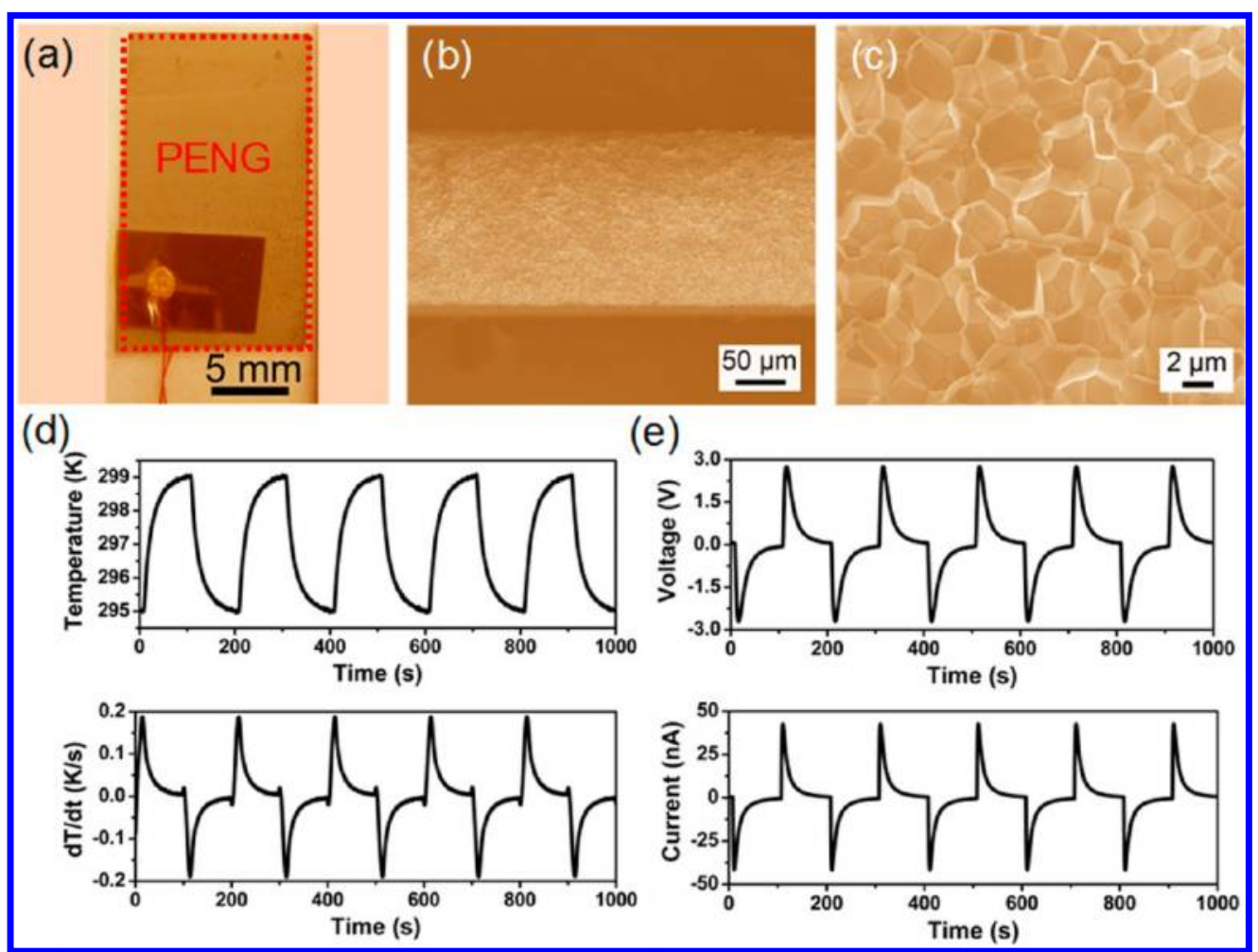

Figure 1. (a) Photograph of a fabricated PENG. (b) Cross-sectional SEM image of the PENG. (c) Enlarged Cross-sectional SEM image of the PENG. (d) The cyclic change in temperature of the PENG and the corresponding differential curve. (e) Measured output voltage and current of the PENG under the change in temperature in $\mathrm{d}$.

1e), and a corresponding positive pulse was received when the temperature was recovered back to $295 \mathrm{~K}$ (Figure 1e). After reversely connecting the PENG to the measurement system, the opposite signals were observed (Figure S1 of the Supporting Information), suggesting that the measured signals were generated by the PENG. Usually, the pyroelectric current $I$ can be described as $I=\mathrm{p} A(\mathrm{~d} T / \mathrm{d} t)$, where $p$ is the pyroelectric coefficient, $A$ is the electrode area, and $\mathrm{d} T / \mathrm{d} t$ is the rate of change in temperature. ${ }^{11}$ According to this equation, the current will linearly increase with increasing the rate of change in temperature, which is consistent with the experimental results, as shown in Figure S2. From the above results, the obtained pyroelectric coefficient of PZT film is about $-80 \mathrm{nC}$ / $\mathrm{cm}^{2} \mathrm{~K}$, which is much larger than that of $\mathrm{ZnO} .^{11,16}$

Figure 2a shows the cyclic change in temperature of PENG from 295 to $340 \mathrm{~K}$ and the corresponding temperature changing rates. The obtained alternating electric output can be rectified by a full-wave bridge circuit, which is needed for driving the polar electronic devices, as shown in Figure 2b. For a temperature change of $45 \mathrm{~K}$, the output voltage and current peaks of PENG are up to $22 \mathrm{~V}$ and $430 \mathrm{nA}$, respectively. The corresponding current density is $171 \mathrm{nA} / \mathrm{cm}^{2}$ by using the surface area of the PENG in Figure 1a. By using the volume of the device in Figure 1a, the corresponding maximum power density is about $0.215 \mathrm{~mW} / \mathrm{cm}^{3}$. Figure $2 \mathrm{c}$ shows an enlarged output voltage peak, which can be used to directly drive a LCD. Usually, the LCD can work under the voltage of larger than 3 $\mathrm{V}$, indicating that the LCD can work in the region of " 2 ", where the working time is longer than $60 \mathrm{~s}$. The attached movie file I (see the Supporting Information) also shows that the LCD can be continuously driven for longer than $60 \mathrm{~s}$, which is much better than that of the previous piezoelectric NG (about $2 \mathrm{~s}$ for an electrical output pulse). ${ }^{17}$
To understand the high output voltage of the PENG in Figure $2 \mathrm{~b}$, we calculated the electrical potential distribution across the PZT film for a temperature change of $45 \mathrm{~K}$ by using COMSOL software. In the calculation, the thickness and the pyroelectric coefficient of the film are $175 \mathrm{~mm}$ and $-80 \mathrm{nC} /$ $\mathrm{cm}^{2} \mathrm{~K}$, respectively. According to the pyroelectric theory, ${ }^{18}$ the change of polarization $\Delta P$ can be given by

$$
\Delta P=p \cdot \Delta T
$$

where $\Delta T$ is the change in temperature. According to the Maxwell equations, ${ }^{19}$ the surface charge density $\sigma$ can be written as

$$
\sigma=\vec{P} \cdot \vec{n}
$$

where $\vec{P}$ is the polarization vector and $\vec{n}$ is the normal vector. In our case, the polarization is uniform and parallel to the direction of normal vector. The surface charge density is only the bound charge density. Thus, the change of surface charge density in our experiment can be given by

$$
\Delta \sigma=\Delta P=p \cdot \Delta T
$$

Along the thickness of the PZT film, the potential $U$ between the top and the bottom electrodes can be expressed as

$$
U=E d=\frac{\Delta \sigma \cdot d}{\left(\varepsilon_{\mathrm{r}}-1\right) \varepsilon_{0}}
$$

where $E$ is the electric field, $d$ is the thickness of PZT film, $\varepsilon_{\mathrm{r}}$ is the relative dielectric constant of the sample, and $\varepsilon_{0}$ is the permittivity of free space. ${ }^{19}$ Using eqs 3 and 4 by COMSOL software package, we can obtain the pyroelectric potential distribution across the PZT film, as shown in Figure 2d. In the calculation, the center along the thickness direction of the PZT 

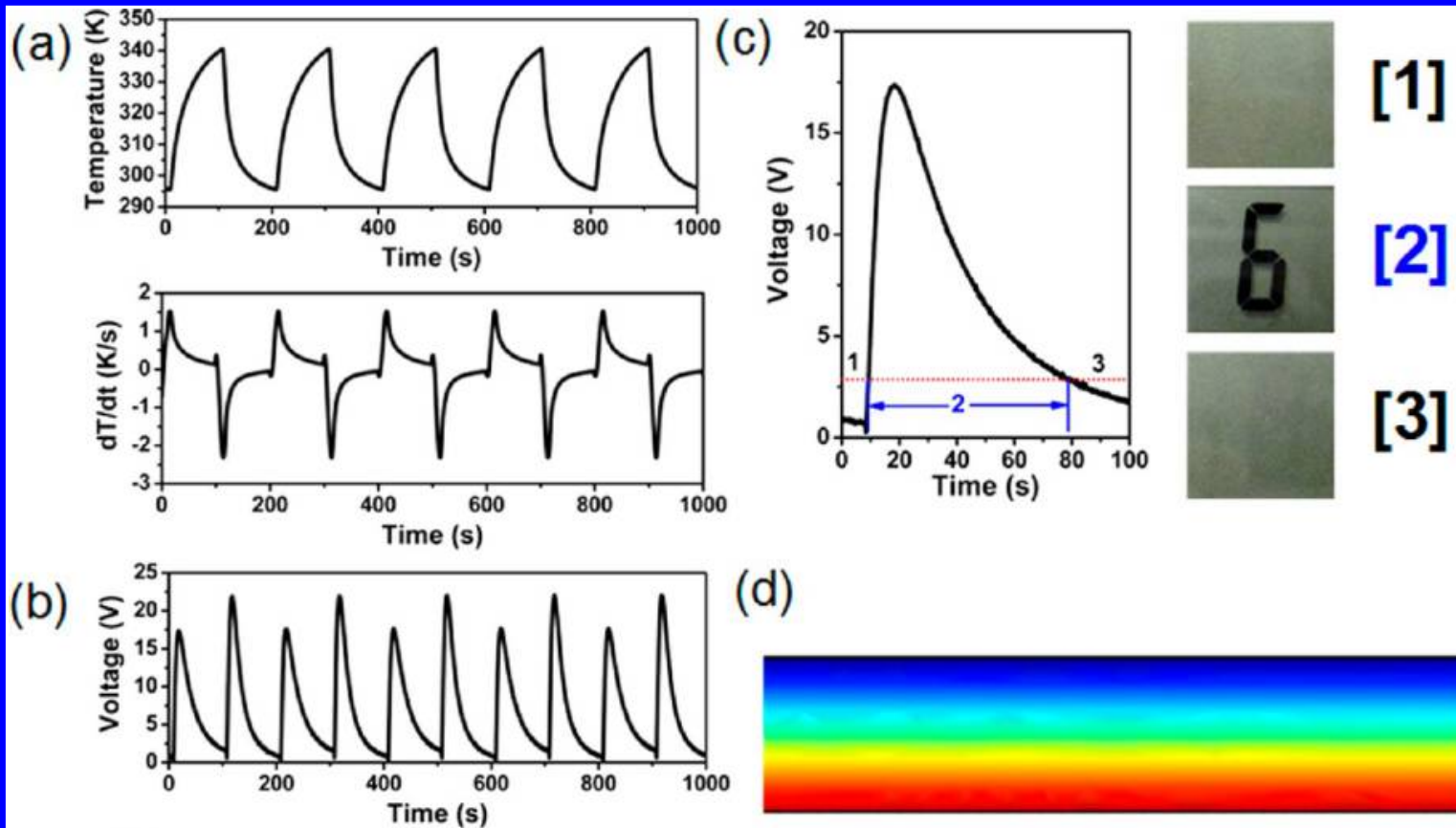

(d)
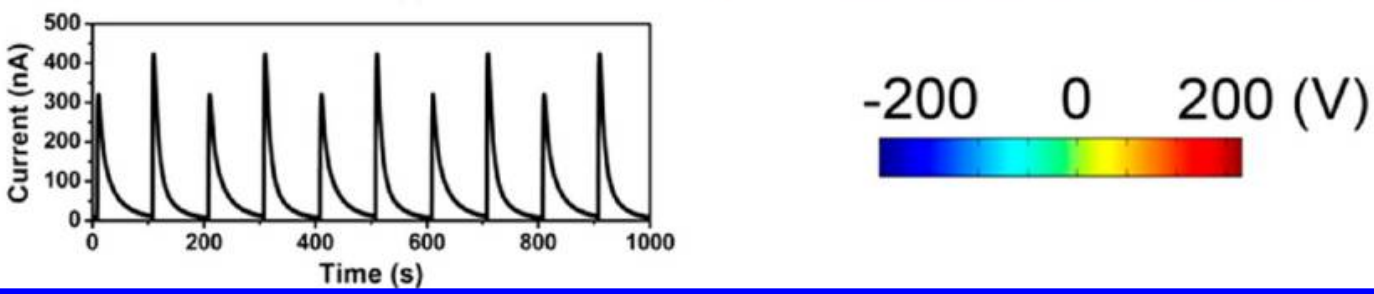

Figure 2. (a) The cyclic change in temperature of the PENG and the corresponding differential curve. (b) Measured output voltage and current of the PENG after they are rectified by a full-wave bridge circuit. (c) Enlarged single output voltage peak, where it can be used to drive a LCD in the region " 2 ". (d) The calculated electrical potential distribution across the PZT film.

film was regarded as the zero potential. The results show that an electrical potential distribution from -200 to $200 \mathrm{~V}$ was created across the film when the temperature was increased from 295 to $340 \mathrm{~K}$. But the measured electrical potential is much lower $(22 \mathrm{~V})$, which means that we have a lot to improve to reach the theoretical value. According to eqs 3 and 4, it can be seen that the pyroelectric potential can be enhanced by increasing the pyroelectric coefficient $p$, the change in temperature $\Delta T$, and the thickness of film $d$.

To expand the potential applications of the PENG, it is necessary to store the electrical energy generated from temperature fluctuation. Figure 3 a shows a schematic diagram that a Li-ion battery was charged by the PENG, which can be used to drive a LED. Figure $3 b$ shows an optical image of the homemade Li-ion coin battery. In such a Li-ion battery, the $\mathrm{TiO}_{2}$ nanotube array grown on $\mathrm{Ti}$ foil was used as the anode. Figure $3 \mathrm{c}$ shows that the diameter of the nanotubes is about $150 \mathrm{~nm}$ and the length is about $10 \mu \mathrm{m}$. The surface of the nanotubes is clean and uniform. The fabrication method of $\mathrm{TiO}_{2}$ nanotubes is given in the Experimental Section. Figure 3d shows the PENG-charging and the subsequent constant-current discharging curves of the battery, where the working frequency of the PENG device is $0.005 \mathrm{~Hz}$ (Figure 2b). The battery can be charged by the PENG from 650 to $810 \mathrm{mV}$ in about $3 \mathrm{~h}$. There are many peaks in the charging curve, which correspond to the output voltage pulses of the PENG in Figure $2 \mathrm{~b}$. The obtained voltage peaks are associated with the universally existing self-discharge of the battery, resulting in that there is no observed voltage platform in the charging curve. The enlarged charging curve is shown in Figure 2d, where the time interval of the two adjacent peaks is about $200 \mathrm{~s}$, which is consistent with that of the PENG in Figure $2 \mathrm{~b}$. The enlarged discharging curve is shown in Figure S3. Under a constant current of $1 \mu \mathrm{A}$, the discharging of the battery lasted for $84 \mathrm{~s}$ before it got back to its original state of $650 \mathrm{mV}$. Thus, the stored electric capacity was about $0.023 \mu \mathrm{Ah}$. To further confirm that the fabricated battery can be charged by the PENG under different conditions, the battery was charged by the PENG at different frequencies $(0.01$ and $0.02 \mathrm{~Hz}$ ), as shown in Figures S4 and S5. It can be found that the time interval of two adjacent peaks is consistent with the working frequency of the device. Figure $3 \mathrm{e}$ shows that the charged Li-ion batteries can be used to power a green LED, which can also be seen in the movie file II (see the Supporting Information).

To illustrate the potential applications of the PENGs, we demonstrated that they may be used as the power sources for wireless sensors. Figure 4a shows a wireless temperature sensor system, which includes the temperature sensor, the signal processing unit, and the flexible antenna. It can be driven by a rechargeable Li-ion battery with a voltage of $2.8 \mathrm{~V}$, as shown in the inset of Figure $4 a$. Figure $4 b$ shows the received signal from the wireless temperature sensor, indicating that the temperature at that time was about $296.2 \mathrm{~K}$. Figure 4c shows the change in temperature in about a half an hour recorded by the wireless temperature sensor with each data for $1 \mathrm{~min}$. The working distance of the wireless sensor can be larger than $50 \mathrm{~m}$. 


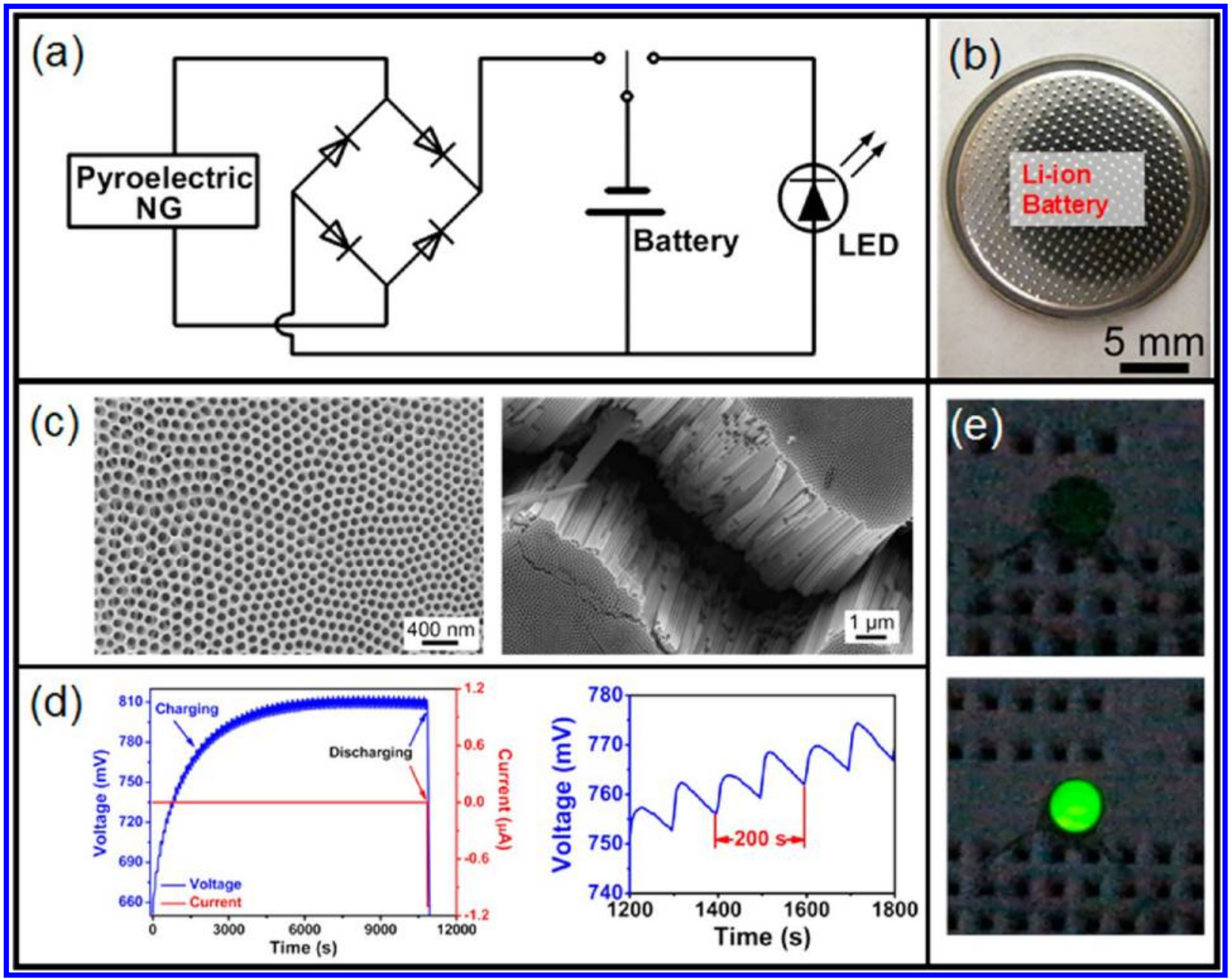

Figure 3. (a) Schematic that illustrates the process for charging the Li-ion battery and driving a LED. (b) Photograph of the fabricated Li-ion battery. (c) SEM image of the obtained $\mathrm{TiO}_{2}$ nanotubes. (d) The charging and discharging curves of the Li-ion battery by using the PENG. (e) Photograph of a green LED driven by the $\mathrm{Li}$-ion batteries.

Currently, the Li-ion battery still cannot be charged to $2.8 \mathrm{~V}$ by the fabricated PENG since the obtained current is not large enough to completely overwhelm the universally existing selfdischarge of the battery. To increase the output current of the PENG, one possible method is to increase the area of the device according to equation of $I=\mathrm{p} A(\mathrm{~d} T / \mathrm{d} t)$. Figure $4 \mathrm{~d}$ shows the output current of a new device is up to $0.8 \mu \mathrm{A}$, which is two times larger than that of the device in Figure $2 \mathrm{~b}$, where the corresponding area is two times larger than that of PENG in Figure $2 \mathrm{~b}$. By using the output voltage and current equations of the PENG, we can find that the increase of both the pyroelectric coefficient and the change in temperature can enhance both the output voltage and the current. Moreover, the output voltage can be improved by increasing the thickness of the PZT film, which has been discussed in eq 4 .

In summary, we have demonstrated the high output pyroelectric nanogenerator based on a PZT film for harvesting thermal energy. Under a temperature change of $45 \mathrm{~K}$ at a rate of $\sim 0.2 \mathrm{~K} / \mathrm{s}$, the pyroelectric output voltage and current density of the PENG are $\sim 22 \mathrm{~V}$ and $\sim 171 \mathrm{nA} / \mathrm{cm}^{2}$, respectively. The single output peak can be used to directly drive a LCD. The theoretical calculation and analysis indicate that the high output performance of the PENG is associated with the large pyroelectric coefficient, the change in temperature, and the thickness of the film. A homemade Li-ion battery can be charged by the PENG under different working frequencies from 0.005 to $0.02 \mathrm{~Hz}$, which can be used to drive a green LED. Our study demonstrates the great potential of PENGs for wireless sensors.

Methods Summary. Fabrication of the PENG and Li-Ion Battery. A 300-nm-thick $\mathrm{Ni}$ layer as the electrode was deposited on both the top and bottom of the PZT film. We applied a high voltage of larger than $4 \mathrm{kV}$ across the PZT film for electric poling at room temperature. Two $\mathrm{Cu}$ wires were used to connect the device and the electrical measurement system, where the $\mathrm{Cu}$ wires were fixed on the surface of the device by the silver paste. A Kapton tape was used to cover the silver paste to keep a good contact between the $\mathrm{Cu}$ wire and the Ni electrode. The $\mathrm{TiO}_{2}$ nanotubes were grown by electrochemically anodizing $\mathrm{Ti}$ foil in ethylene glycol solution, ${ }^{20,21}$ which was used as the anode material of the Li-ion battery. The cathodes are $\mathrm{LiCoO}_{2} /$ conductive carbon/binder mixtures on aluminum foils. The polyethylene film was used as the separator material.

Measurement of the PENG, Li-lon Battery, and Wireless Temperature Sensor. The output voltage of the PENG was measured by a low-noise voltage preamplifier $(<3 \mathrm{~V}$, Stanford Research SR570; >3 V, Keithley 6514 system electrometer). The output current of the PENG was measured by a low-noise current preamplifier (Stanford Research SR560). A heater was 


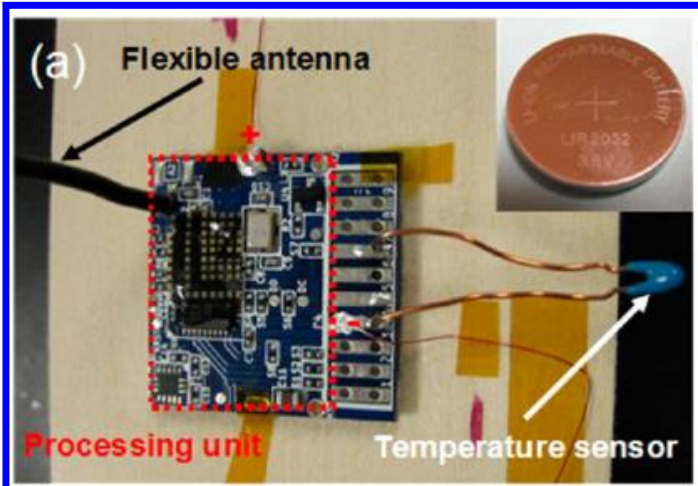

(b)
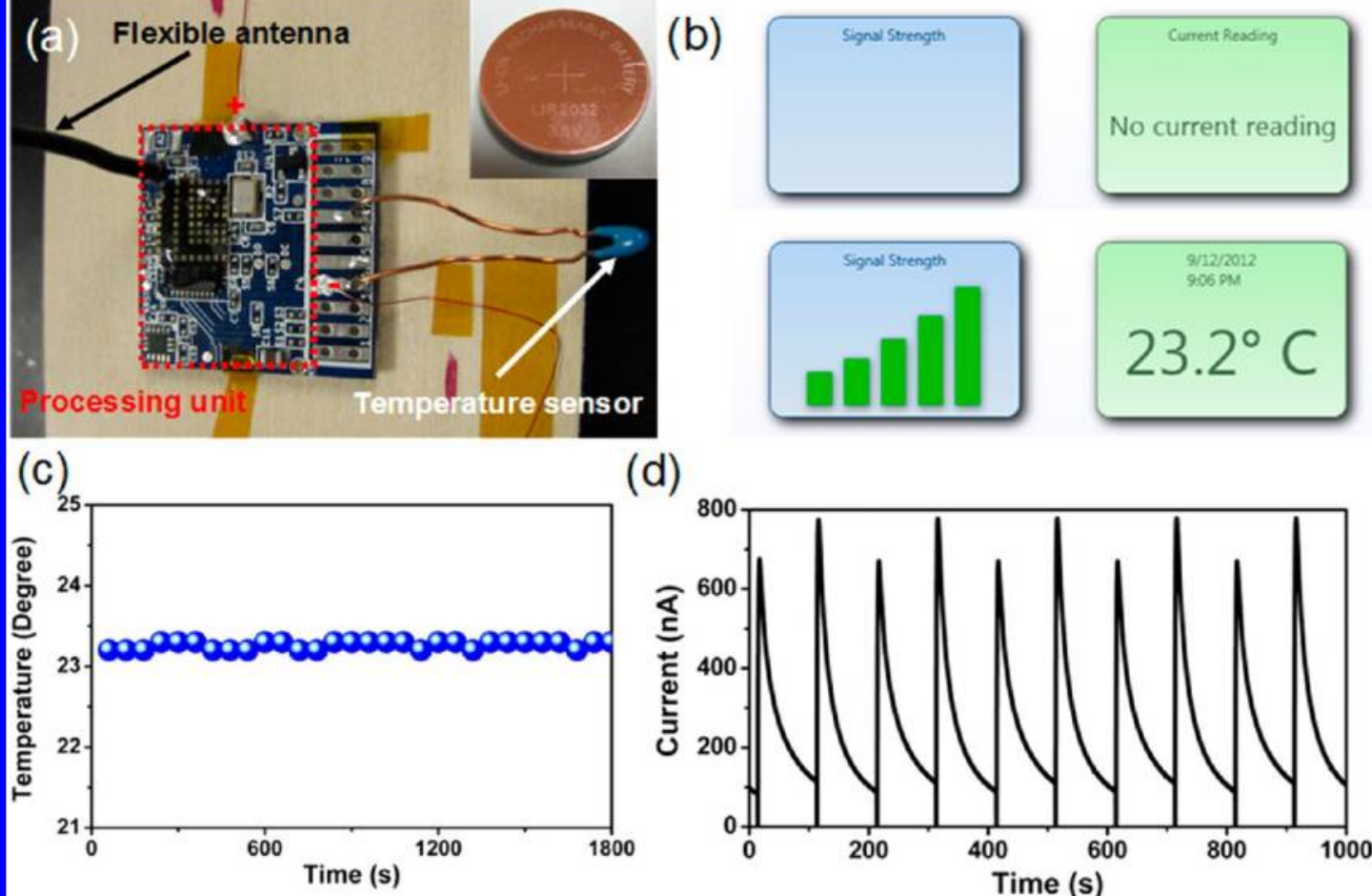

(d)

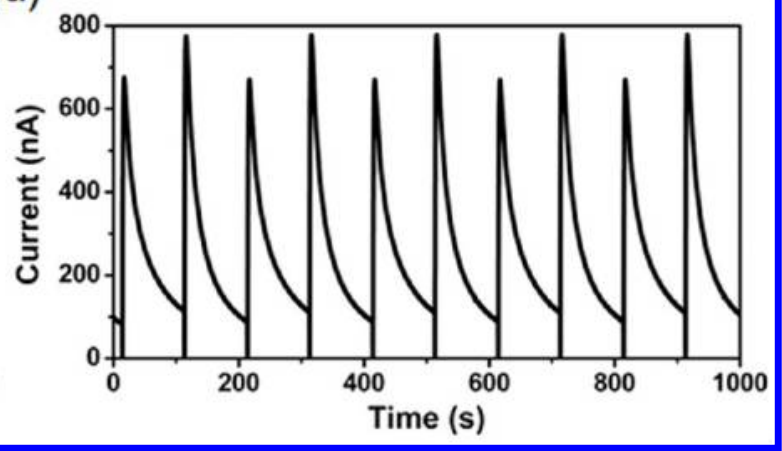

Figure 4. (a) Photograph of a wireless temperature sensor. The inset shows the photograph of a rechargeable Li-ion battery. (b) The obtained temperature signals when the wireless temperature sensor was turned off and on. (c) The obtained temperature data in half an hour, where the temperature value was recorded in every $1 \mathrm{~min}$. (d) Output current of the new PENG when the surface area of the device was increased to 2 times as compared with that in Figure $2 b$.

used to apply a cyclic change in temperature of the PENGs, where a temperature sensor was used to detect the change in temperature of the PENGs. The performance of the Li-ion battery was measured by a battery analyzer (MTI Corporation). There are eight channels, which can be used to measure eight batteries at the same time. The used wireless temperature sensor has an accurate of $\pm 1 \mathrm{~K}$, and the working distance is larger than $50 \mathrm{~m}$, which can be used for environmental temperature monitoring.

\section{ASSOCIATED CONTENT}

\section{S Supporting Information}

Additional figures about the output voltage/current under the reversed connection to the measured system, the output current under the different temperature changing rates, the enlarged discharging curves in Figure $3 \mathrm{~d}$, the charging and discharging processes when the working frequencies of the PENG are 0.01 and $0.02 \mathrm{~Hz}$. The additional movie files include that the first one (I) is to drive a LCD by using a single output pulse, and the second one (II) is to drive a LED by using the Li-ion batteries. This material is available free of charge via the Internet at http://pubs.acs.org.

\section{AUTHOR INFORMATION}

\section{Corresponding Author}

*E-mail: zlwang@gatech.edu.

\section{Author Contributions}

$\S$ These authors contributed equally to the work.

\section{Notes}

The authors declare no competing financial interest.

\section{ACKNOWLEDGMENTS}

This work was supported by Airforce, MURI, U.S. Department of Energy, Office of Basic Energy Sciences (DE-FG0207ER46394), NSF, and the Knowledge Innovation Program of the Chinese Academy of Sciences (KJCX2-YW-M13).

\section{REFERENCES}

(1) Wang, Z. L. Adv. Mater. 2011, 82, 279-284.

(2) Dong, Y.; Tian, B.; Kempa, T. J.; Lieber, C . M. Nano Lett. 2009, 9, 2183-2187.

(3) Hu, L.; Choi, J. W.; Yang, Y.; Jeong, S.; Mantia, F. L.; Cui, L.-F.; Cui, Y. Proc. Natl. Acad. Sci. U.S.A. 2009, 106, 21490-21494.

(4) Qi, Y.; Jafferis, N. T.; Lyons, K., Jr.; Lee, C. M.; Ahmad, H.; McAlpine, M. C. Nano Lett. 2010, 10, 524-528.

(5) Chang, C.; Tran, V. H.; Wang, J.; Fuh, Y.-K.; Lin, L. Nano Lett. 2010, 10, 726-731.

(6) Fan, F.-R.; Tian, Z.-Q.; Wang, Z. L. Nano Energy 2012, 1, 328334.

(7) Zhu, G.; Pan, C.; Guo, W.; Chen, C.-Y.; Zhou, Y.; Yu, R.; Wang, Z. L. Nano Lett. 2012, 12, 4960-4965.

(8) Lang, S. B. Phys. Today 2005, 58, 31-36.

(9) Yang, Y.; Pradel, K. C.; Jing, Q.; Wu, J. M.; Zhang, F.; Zhou, Y.; Zhang, Y.; Wang, Z. L. ACS Nano 2012, 6, 6984-6989.

(10) Liu, W.; Yan, X.; Chen, G.; Ren, Z. Nano Energy 2012, 1, 4256.

(11) Yang, Y.; Guo, W.; Pradel, K. C.; Zhu, G.; Zhou, Y.; Zhang, Y.; Hu, Y.; Lin, L.; Wang, Z. L. Nano Lett. 2012, 12, 2833-2838.

(12) Xu, S.; Qin, Y.; Xu, C.; Wei, Y.; Yang, R.; Wang, Z. L. Nat. Nanotechnol. 2010, 5, 366-373.

(13) Zhu, J.; Hsu, C.-M.; Yu, Z.; Fan, S.; Cui, Y. Nano Lett. 2010, 10, 1979-1984.

(14) Lee, M.; Bae, J.; Lee, J.; Lee, C.-S.; Hong, S.; Wang, Z. L. Energy Environ. Sci. 2011, 4, 3359-3363. 
(15) Yang, Y.; Jung, J. H.; Yun, B. K.; Zhang, F.; Pradel, K. C.; Guo, W.; Wang, Z. L. Adv. Mater. 2012, 24, 5357-5362.

(16) Ye, C.-P.; Tamagawa, T.; Polla, D. L. J. Appl. Phys. 1991, 70, $5538-5543$.

(17) Hu, Y.; Xu, C.; Zhang, Y.; Lin, L.; Snyder, R. L.; Wang, Z. L. Adv. Mater. 2011, 23, 4068-4071.

(18) Zook, J. D.; Liu, S. T. J. Appl. Phys. 1978, 49, 4604-4606.

(19) Grant, I. S.; Phillips, W. R. Electromagnetism, 2nd ed.; Manchester Physics, John Wiley \& Sons: New York, 2008.

(20) Ye, M. D.; Xin, X. K.; Lin, C. J.; Liu, Z. Q. Nano Lett. 2011, 11, $3214-3220$.

(21) Xue, X.; Wang, S.; Guo, W.; Zhang, Y.; Wang, Z. L. Nano Lett. 2012, 12, 5048-5054. 